\title{
HIV-1 GENOTYPIC DRUG RESISTANCE TESTING: DIGGING DEEP, REACHING WIDE?
}

\author{
Kristel Van Laethem ${ }^{1,2}$, Kristof Theys ${ }^{1}$, Anne-Mieke Vandamme $e^{1,3}$ \\ ${ }^{1}$ KU Leuven - University of Leuven, Department of Microbiology and Immunology, Rega Institute for \\ Medical Research, Clinical and Epidemiological Virology, Minderbroedersstraat 10, B-3000 Leuven, \\ Belgium \\ ${ }^{2}$ University Hospitals Leuven, AIDS Reference Laboratory, Herestraat 49, 3000 Leuven, Belgium \\ ${ }^{3}$ Global Health and Tropical Medicine and Unidade de Microbiologia, Instituto de Higiene e Medicina \\ Tropical, Universidade Nova de Lisboa, Lisbon, Portugal
}

Corresponding author: Kristel Van Laethem, kristel.vanlaethem@uzleuven.be, tel: +32-16332177

\begin{abstract}
For many years, population-based Sanger sequencing has been the golden standard for drug resistance testing within the routine follow-up of HIV-1 infected patients in resource-rich settings. Often, the data generated within this framework were subsequently used for research and surveillance purposes: to understand therapy response and to gain insights into epidemiological processes. Sanger sequencing was however ill suited for diagnostic and prognostic use in resourcelimited settings (RLS) and therefore not broadly implemented. Next-generation sequencing (NGS) technologies provide high-throughput approaches by the rapid acquisition of thousands to millions of short nucleotide sequences. Depending on the experimental design, the roll-out of NGS drug resistance testing at a larger scale is feasible, providing better characterization and understanding of the evolving population of viral variants within a patient and potentially improving the prognostic value of drug resistance testing. Whether the same will become true for RLS will largely depend on affordability and sample logistics, and this may affect other mutation-specific approaches.
\end{abstract}




\section{Introduction}

Combination antiretroviral therapy (CART) has significantly improved survival rates and quality of life of patients infected with the Human Immunodeficiency Virus type 1 (HIV-1). However, lifelong treatment and medical follow-up are required as a therapeutic cure remains elusive, while host-, virus- and drug-related suboptimal treatment and adherence are accompanied with emerging drug resistance and subsequent transmission of drug-resistant strains which jeopardize the advancements in HIV-1 care [1].

In particular, the high evolutionary rate of HIV-1, driven by the high mutation rate during reverse transcription, results into a heterogeneous viral population within HIV-1 patients [2]. When virus replication cannot be shut down during treatment, the competitive advantage of a minority variant with higher fitness under drug selective pressure will result in an increase of its frequency ultimately allowing resistance mutations to accumulate and treatment to fail.

Since many years, drug resistance testing has enabled personalized strategies for the treatment of HIV-1 infected patients in resource-rich settings (RRS) (Table 1) [3]. At diagnosis and entry into care, such testing is to uncover transmitted drug resistance (TDR) while at virological failure, causality assessment of failure is the goal. In both instances, the detected mutation profile guides the clinician in the subsequent selection of a potent cART, although current methods have limitations in explaining and predicting therapeutic outcome [4]. In the absence of simple and affordable resistance tests, resource-limited settings (RLS) opted for a public health approach with the implementation of drug resistance surveillance at sentinel sites to guide the decisions on countrywide CART programs [5].

Recent technical innovations may enable the roll-out of new and powerful genotypic testing in both RSS and RLS and contribute to a more comprehensive assessment of the emergence and spread of drug resistance and how to maintain long-term suppressive cART. Implementation of the various strategies should however be preceded by an evaluation of their performance in the context of a changing HIV epidemic.

\section{Population-based Sanger sequencing as current golden standard for drug resistance testing in clinical virology}

For many years, routine monitoring of drug resistance is predominantly done using population-based Sanger sequencing (PBSS) due to its superior characteristics compared to phenotypic resistance assays and mutation-specific assays. Although the interpretation of complex mutational patterns into clinically relevant cut-offs is not straightforward, genotyping is characterized with shorter turnaround times, lower cost, greater accessibility and an earlier detection of emerging drug resistance than phenotyping (Table 2) [6]. In comparison to Line Probe Assay (LiPA), a technology that displayed a higher analytical sensitivity for minority variants and enabled the simultaneous investigation of several mutations, PBSS provided more complete information on drug resistance and proved to be more reliable [7].

\section{Next-generation sequencing technologies}

Several next-generation sequencing (NGS) technologies are currently being explored for HIV drug resistance testing [8]. The 454-pyrosequencing method (Roche) used to be the preferred technology because of its longer read lengths (400-700bp), but uncompetitive prices and frequency of indel errors ( 1\%) recently resulted in its discontinuation. These errors were especially detrimental for HIV-1 drug resistance testing as they mainly occur in homo-polymer stretches which are commonly found within or adjacent to HIV-1 codons associated with drug resistance [9]. Although suffering from similar problems inherent to the pyrosequencing methodology, lon Torrent partially circumvents the problem through template barcoding and bioinformatics pipelines and is still clinging to the market because of its highly competitive pricing and shorter run times. The main player in the field is currently Illumina, given its simple work flow and lower rate of systematic errors $(\sim 0.1 \%$, substitutions), although long run times and shorter read lengths (125-250bp) are still disadvantages. While local reconstruction of minority variants can identify the co-occurrence of resistance 
mutations in a single variant when their genomic distance is lower than the average read length, inferring larger sequence fragments of minority variants is challenged by the short read lengths and high error rates $[10,11]$. The latest innovative technologies that could be a major improvement in this respect are Pacific Biosciences and Oxford Nanopore Technology that both enable single molecule, real-time DNA sequencing of extensive read length $(\geq 1000 \mathrm{bp})$. Their breakthrough is still pending due to their high error rates ( $\geq 12 \%$ at single runs) and costs.

\section{Changing scenes in antiviral therapy and drug resistance}

Since a few years, the prevalence of virological treatment failure and acquired drug resistance have been declining significantly in RRS [12]. These recent successes are mainly attributed to the introduction of new drug classes and to more potent, tolerable and convenient drugs within existing classes, as well as to resistance-guided therapy.

Despite this decline in acquired resistance, levels of TDR have remained relatively stable over 19992013 although TDR rates and trends varied by geographical region, risk group and drug class [13]. An in-depth analysis demonstrated that TDR slightly increased during times lacking access to new drugs and only sharply decreased upon the introduction of a new potent drug class, such as boosted protease and integrase inhibitors, keeping the overall TDR trend stable [14]. This is a potential cause of concern when drug pipelines dry up. Therefore, TDR will remain a problem to be monitored.

By contrast, drug stock-outs and insufficient laboratory monitoring still challenge the long-term success of CART in RLS. Several studies have shown increasing levels of transmitted and acquired drug resistance in these regions [15-16]. As non-B subtypes prevail and other drug combinations than the ones used in RRS have been used, drug-selected amino acid substitution patterns might be different and their impact on therapy outcome unclear $[17,18]$. The need for drug resistance testing is thus increasing in RLS.

\section{Ultra-wide next-generation sequencing for genotypic drug resistance testing}

With Trugene HIV-1 Genotyping Assay (Siemens) abandoned, ViroSeq HIV-1 Genotyping System (Abbott Molecular) is the only FDA-cleared and CE marked propriety system for in vitro diagnostic use of drug resistance testing. As a result, many virology laboratories have switched to in-house methods to address the need for simultaneous drug resistance testing against all six drug classes. They require more extensive optimization, standardization and validation efforts than commercial assays (Table 2) [19]. Furthermore, with an ever larger number of genetic regions becoming clinically relevant, PBSS costs and turnaround times are rising steadily.

NGS may bridge this gap, as it could enable the reliable sequencing of more extended and even problematic (by PBSS methods) genetic regions (Figure 1) (Table 2) [20,21]. Ultra-wide sequencing of the full-length viral genome, generating additional information on amino acid substitutions in Gag, Pol and Env, could enhance therapeutic outcome predictions [22-27].

For the required template enrichment, the current full-genome protocols rely on amplification of large genomic regions with subtype-wide primers and therefore perform less well at viral loads below 1000-3000 copies/ml, making these assays currently more appropriate for surveillance efforts than for monitoring of individual patients $[28,29]$. However, several studies have already shown that experienced laboratories can achieve accurate NGS results at frequencies as low as 1\% [30-33]. Although sequencing reagents cost per sample drops significantly when multiplexing is considered, this up-scaling requires more experimental steps and extensive in-silico analyses which increase direct and indirect costs, risk for human error and turn-around times (Table 2).

\section{Far-reaching innovations: mutation-specific assays versus next-generation sequencing for} surveillance in resource-limited settings

In view of the paucity of PBSS for routine monitoring in RLS, mutation-specific assays have been developed to meet the need for large-scale drug resistance surveillance at a reasonable cost $[34,35]$. These assays are based upon selective amplification, hybridization or ligation at only a limited 
number of pre-defined sentinel codons, but with the advantage of detecting minority variants below the threshold of PBSS (Table 2) [36].

Although mutation-specific assays are considered to be easy to perform and therefore suitable for implementation at local sites, experience with the specific methodology were important factors for achieving valid results [37]. In addition, primers and probes needed to be modified to the locally circulating viral variant of a subtype or, more extremely, to the patient-specific consensus sequence to decrease the number of indeterminate and inaccurate results [37-39]. It seems the problems formerly experienced by LiPA have not all been solved.

Therefore, other research teams opted for the implementation of more complex NGS methods in centralized national or regional laboratories for the surveillance of drug resistance and the investigation of transmission dynamics [40].

\section{Mutation-specific assays versus next-generation sequencing for sensitive detection of drug resistance}

Advances in resistance genotyping for monitoring individual patients are expected to come from a better knowledge of the impact of minority resistant variants. Studies have demonstrated that the use of mutation-specific assays and ultra-deep sequencing results in higher drug resistance levels in therapy-naïve and -experienced patients compared to PBSS [41-49].

However, this effect was strongly depended on the number of mutations evaluated, the experimental set-up (detection cut-off used), the clinical context (drug exposure [50,51], time elapse since infection or drug exposure [52,53]), and the specific drugs or mutations evaluated $[54,55]$.

The transmission dynamics within a population and the fitness cost of a particular mutation affects the relevance of more sensitive testing. In RRS, the rarely observed M184V in recently-infected patients is most often transmitted from patients failing virologically, even though therapy-naïve patients seem to be the major source of new infections and therefore of TDR [56]. M184V has a high fitness cost, and thus its reversal and gradually decay in a drug-free environment prevents its onward transmission, in contrast to low fitness cost drug resistance mutations, such as M41L, T215 revertants and $\mathrm{K} 103 \mathrm{~N}$ [57]. This might explain why sensitive testing does not always result in higher TDR rates in chronically infected patients [58].

\section{Predictive value of ultra-deep next-generation sequencing for therapeutic response}

In recent years, studies have suggested that drug resistant variants not detected by PBSS may influence the response to first-line cART [59]. Consequently, mutation-specific assays and NGS methods have been used to determine the impact of drug-resistant minority variants on therapeutic outcome in several retrospective observational studies.

The relevance of testing for minority variants has been shown primarily for maraviroc and nonnucleoside reverse transcriptase inhibitors (NNRTI). When retrospectively reanalyzing patients treated with maraviroc-containing CART, prediction of treatment outcome was improved when using assays with enhanced sensitivity for CXCR4-using variants [60-62]. Although the presence of CXCR4using variants was associated with an increased risk of therapeutic failure, yet, the superiority of ultra-deep sequencing over PBSS has not been shown.

HIV-1 patients with NNRTI-resistant variants that could only be detected as minority variants show a more than 2-fold higher risk of virologically failing an NNRTI-containing first-line regimen. In some studies, a dose effect of the frequency or mutational load was shown $[63,64]$. The clinical relevance of NNRTI resistant minority variants has not consistently been confirmed, and has not been demonstrated for other first-line regimens, such as those containing integrase or boosted protease inhibitors, therefore guidance on the use of deep sequencing is still lacking.

In the context of virological failure, an improved predictive performance for success of salvage regimen was shown by Pou et al. in a retrospective analysis, while Charpentier et al. failed to demonstrate a similar effect $[54,55,65]$. An added complexity is that current NGS methods need viral loads that are higher than usually found at treatment failure to reliable detect minority variants. 
Although sensitive methods possess a great potential in improving the prognostic value of drug resistance testing, to date, technical improvements and a better understanding of the impact of minority resistant variants are required before proceeding to clinical studies, sufficiently powered for the assessment of ultra-deep sequencing as a prognostic tool.

\section{Digging deep and reaching wide}

In contrast to mutation-specific assays that are limited to the detection and quantification of particular drug resistance mutations, NGS has the potential to reconstruct the viral population by identifying the set of individual virus variants with their respective frequency or mutational load (ultra-deep and ultra-wide sequencing). Information on the co-occurrence of drug resistance and accessory mutations at the level of individual viral variants could lead to a better understanding of viral escape dynamics from therapeutic pressure. By distinguishing minority variants with single drug resistance mutations that were merely the result of random genetic variation from variants that have subsequently further adapted to therapeutic pressure, NGS could potentially lead to more accurate predictions of therapeutic response [51].

\section{Conclusion}

The decision whether new technologies are fit for HIV-1 drug resistance testing and can transit from research purposes to clinical and epidemiological settings will largely depend on the strength of findings from large studies. High-throughput technologies can clearly contribute significantly to more extensive surveillance of drug resistance globally. Although, consistent results that could support the prognostic use of ultra-deep sequencing for individual patients are lacking to date, routine follow-up by ultra-wide sequencing with a reporting limit set at depths similar to PBSS might be a pragmatic approach and readily implemented (Figure 1). From such efforts, continuous growth of data and knowledge will speed up the development of standardized drug resistance interpretation systems and guidance documents for the clinical implementation of NGS, as has been done for PBSS $[3,66]$. An eventual introduction into clinical practice will ultimately depend on specifications and performance characteristics (Table 2). The biggest challenges relate to wet-lab automation and a bioinformatics pipeline [11], which are not straightforward for many virology laboratories. These hurdles could be partly surpassed by centralizing HIV drug resistance testing or partnering with genomics core centers, and the development of user-friendly software enabling a standardized approach for the reconstruction of minority variants, the detection of drug resistance and the prediction of therapeutic outcome.

\section{Acknowledgements}

This work was partly supported by Fonds voor Wetenschappelijk Onderzoek Vlaanderen (G.06.92.14N, PDO/11), by the University of Leuven (PF/10/018;) and by the European Union (Horizon2020 research and innovation programme, Virogenesis grant $\mathrm{nr} 634650$ ). KT receives a FWO postdoctoral fellowship. The funders had no role in this study.

\section{References}

Papers of particular interest, published within the period of review, have been highlighted as:

* Of special interest

** of outstanding interest

1. Paredes R, Clotet B: Clinical management of HIV-1 resistance. Antiviral Res 2010, 85:245-265.

2. Domingo E, Sheldon J, Perales C: Viral quasispecies evolution. Microbiol Mol Biol Rev 2012, 76:159-216.

** An outstanding review on the theory of viral quasispecies and the biological implications of quasispecies dynamics. 
3. Vandamme AM, Camacho RJ, Ceccherini-Silberstein F, de Luca A, Palmisano L, Paraskevis D, Paredes R, Poljak M, Schmit JC, Soriano V et al.: European recommendations for the clinical use of HIV drug resistance testing: 2011 update. AIDS Rev 2011, 13:77-108.

4. Frentz D, Boucher CA, Assel M, De Luca A, Fabbiani M, Incardona F, Libin P, Manca N, Müller V, O Nualláin $B$ et al.: Comparison of HIV-1 genotypic resistance test interpretation systems in predicting virological outcomes over time. PLoS One 2010, 5:e11505.

5. Gilks CF, Crowley S, Ekpini R, Gove S, Perriens J, Souteyrand Y, Sutherland D, Vitoria M, Guerma T, De Cock $\mathrm{K}$. The WHO public-health approach to antiretroviral treatment against HIV in resource-limited settings. Lancet 2006, 368:505-510.

6. Van Laethem K, Van Vaerenbergh K, Schmit JC, Sprecher S, Hermans P, De Vroey V, Schuurman R, Harrer T, Witvrouw $M$, Van Wijngaerden $E$ et al.: Phenotypic assays and sequencing are less sensitive than point mutation assays for detection of resistance in mixed HIV-1 genotypic populations. J Acquir Immune Defic Syndr 1999, 22:107-118.

7. Koch N, Yahi N, Colson P, Fantini J, Tamalet C: Genetic polymorphism near HIV-1 reverse transcriptase resistance-associated codons is a major obstacle for the line probe assay as an alternative method to sequence analysis. J Vir Methods 1999, 80:25-31.

8. Quiñones-Mateu ME, Avila S, Reyes-Teran G, Martinez MA. Deep sequencing: becoming a critical tool in clinical virology. J Clin Virol 2014, 61:9-19.

* A review on the use of current next-generation sequencing platforms in clinical virology.

9. Dudley DM, Chin EN, Bimber BN, Sanabani SS, Tarosso LF, Costa PR, Sauer MM, Kallas EG, O'Connor DH: Low-cost ultra-wide genotyping using Roche/454 pyrosequencing for surveillance of HIV drug resistance. PLoS One 2012, 7:e36494.

10. Vrancken $B$, Lequime $S$, Theys $K$, Lemey $P$.: Covering all bases in HIV research: unveiling a hidden world of viral evolution. AIDS Rev 2010, 12:89-102.

11. Beerenwinkel N, Günthard HF, Roth V, Metzner KJ: Challenges and opportunities in estimating viral genetic diversity from next-generation sequencing data. Front Microbiol 2012, 3:329.

** An excellent review on the experimental, statistical and computational challenges of nextgeneration sequencing.

12. De Luca A, Dunn D, Zazzi M, Camacho R, Torti C, Fanti I, Kaiser R, Sönnerborg A, Codoñer FM, Van Laethem $\mathrm{K}$ et al.: Declining prevalence of HIV-1 drug resistance in antiretroviral treatmentexposed individuals in Western Europe. $J$ Infect Dis 2013, 207:1216-1220.

13. Pham QD, Wilson DP, Law MG, Kelleher AD, Zhang L: Global burden of transmitted HIV drug resistance and HIV-exposure categories: a systematic review and meta-analysis. AIDS 2014, 28:2751-2762.

14. Yang WL, Kouyos R, Scherrer AU, Böni J, Shah C, Yerly S, Klimkait T, Aubert V, Furrer H, Battegay $M$ et al.: Assessing the paradox between transmitted and acquired HIV type 1 drug resistance mutations in the Swiss HIV Cohort Study from 1998 to 2012. J Infect Dis 2015.

15. Gupta RK, Jordan MR, Sultan BJ, Hill A, Davis DH, Gregson J, Sawyer AW, Hamers RL, Ndembi N, Pillay $D$, Bertagnolio $S$ : Global trends in antiretroviral resistance in treatment-naive individuals with HIV after rollout of antiretroviral treatment in resource-limited settings: a global collaborative study and meta-regression analysis. Lancet 2012, 380:1250-1258.

16. Ssemwanga D, Lihana RW, Ugoji C, Abimiku A, Nkengasong J, Dakum P, Ndembi N: Update on HIV-1 acquired and transmitted drug resistance in Africa. AIDS Rev 2015, 17:3-20.

17. Megens S, Van Laethem K: HIV-1 genetic variation and drug resistance development. Expert Rev Anti Infect Ther 2013, 11:1159-1178.

18. Wainberg MA, Brenner BG: The impact of HIV genetic polymorphisms and subtype differences on the occurrence of resistance to antiretroviral drugs. Mol Biol Int 2012, 2012:256982.

19. Alemán Y, Vinken L, Kourí V, Pérez L, Álvarez A, Abrahantes Y, Fonseca C, Pérez J, Correa C, Soto Y et al.: Performance of an in-house human immunodeficiency virus type 1 genotyping system for assessment of drug resistance in Cuba. PLoS One 2015, 10:e0117176. 
20. Van Laethem $K$, Schrooten $Y$, Lemey $P$, Van Wijngaerden $E$, De Wit $S$, Van Ranst $M$, Vandamme AM: A genotypic resistance assay for the detection of drug resistance in the human immunodeficiency virus type 1 envelope gene. J Virol Methods 2005, 123:25-34.

21. Van Laethem K, Schrooten Y, Dedecker S, Van Heeswijck L, Deforche K, Van Wijngaerden E, Van Ranst M, Vandamme AM: A genotypic assay for the amplification and sequencing of gag and protease from diverse human immunodeficiency virus type 1 group $M$ subtypes. J Virol Methods 2006, 132:181-186.

22. Brehm JH, Koontz DL, Wallis CL, Shutt KA, Sanne I, Wood R, McIntyre JA, Stevens WS, SluisCremer N, Mellors JW, CIPRA-SA Project 1 Study Team: Frequent emergence of N348I in HIV-1 subtype $C$ reverse transcriptase with failure of initial therapy reduces susceptibility to reversetranscriptase inhibitors. Clin Infect Dis 2012, 55:737-745.

23. Thielen A, Sichtig N, Kaiser R, Lam J, Harrigan PR, Lengauer T: Improved prediction of HIV-1 coreceptor usage with sequence information from the second hypervariable loop of gp120. $J$ Infect Dis 2010, 202:1435-1443.

24. Fun A, Wensing AM, Verheyen J, Nijhuis M: Human Immunodeficiency Virus Gag and protease: partners in resistance. Retrovirology 2012, 9:63.

25. Rabi SA, Laird GM, Durand CM, Laskey S, Shan L, Bailey JR, Chioma S, Moore RD, Siliciano RF: Multi-step inhibition explains HIV-1 protease inhibitor pharmacodynamics and resistance. $J$ Clin Invest 2013, 123:3848-3860.

26. Sutherland KA, Ghosn J, Gregson J, Mbisa JL, Chaix ML, Cohen Codar I, Delfraissy JF, Delaugerre C, Gupta RK: HIV-1 subtype influences susceptibility and response to monotherapy with the protease inhibitor lopinavir/ritonavir. J Antimicrob Chemother 2015, 70:243-248.

27. Zhang XM, Wu H, Zhang Q, Lau TC, Chu H, Chen ZW, Jin DY, Zheng BJ: A novel mutation, D404N, in the connection subdomain of reverse transcriptase of HIV-1 CRF08_BC subtype confers cross-resistance to NNRTIs. J Antimicrob Chemother 2015, 70:1381-1390.

28. Gall A, Ferns B, Morris C, Watson S, Cotten M, Robinson M, Berry N, Pillay D, Kellam P: Universal amplification, next-generation sequencing, and assembly of HIV-1 genomes. J Clin Microbiol 2012, 50:3838-3844.

29. Dudley DM, Bailey AL, Mehta SH, Hughes AL, Kirk GD, Westergaard RP, O'Connor DH: Cross-clade simultaneous HIV drug resistance genotyping for reverse transcriptase, protease, and integrase inhibitor mutations by Illumina MiSeq. Retrovirology 2014, 11:122.

30. Avidor B, Girshengorn S, Matus N, Talio H, Achsanov S, Zeldis I, Fratty IS, Katchman E, BroshNissimov $T$, Hassin $D$ et al.: Evaluation of a benchtop HIV ultradeep pyrosequencing drug resistance assay in the clinical laboratory. J Clin Microbiol 2013, 51:880-886.

31. Gibson RM, Meyer AM, Winner D, Archer J, Feyertag F, Ruiz-Mateos E, Leal M, Robertson DL, Schmotzer CL, Quiñones-Mateu ME: Sensitive deep-sequencing-based HIV-1 genotyping assay to simultaneously determine susceptibility to protease, reverse transcriptase, integrase, and maturation inhibitors, as well as HIV-1 coreceptor tropism. Antimicrob Agents Chemother 2014, 58:2167-2185.

* Very informative study on the validation of an in-house next-generation assay.

32. Knapp DJ, McGovern RA, Poon AF, Zhong X, Chan D, Swenson LC, Dong W, Harrigan PR: "Deep" sequencing accuracy and reproducibility using Roche/454 technology for inferring co-receptor usage in HIV-1. PLoS One 2014, 9:e99508.

33. Simen BB, Braverman MS, Abbate I, Aerssens J, Bidet Y, Bouchez O, Gabriel C, Izopet J, Kessler $\mathrm{HH}$, Stelzl $\mathrm{E}$ et al.: An international multicenter study on HIV-1 drug resistance testing by $\mathbf{4 5 4}$ ultra-deep pyrosequencing. J Virol Methods 2014, 204:31-37.

* External quality assessment of next-generation sequencing assays.

34. Gianella S, Richman DD: Minority variants of drug-resistant HIV. J Infect Dis 2010, 202:657-666.

35. Zhang G, Cai F, Zhou Z, DeVos J, Wagar N, Diallo K, Zulu I, Wadonda-Kabondo N, Stringer JS, Weidle PJ et al.: Simultaneous detection of major drug resistance mutations in the protease and reverse transcriptase genes for HIV-1 subtype $\mathrm{C}$ by use of a multiplex allele-specific assay. $J$ Clin Microbiol 2013, 51:3666-3674. 
36. Halvas EK, Aldrovandi GetM, Balfe P, Beck IA, Boltz VF, Coffin JM, Frenkel LM, Hazelwood JD, Johnson VA, Kearney $M$ et al.: Blinded, multicenter comparison of methods to detect a drugresistant mutant of human immunodeficiency virus type 1 at low frequency. J Clin Microbiol 2006, 44:2612-2614.

37. Beck IA, Crowell C, Kittoe R, Bredell H, Machaba M, Willamson C, Janssens W, Jallow S, van der Groen $G$, Shao $Y$ et al.: Optimization of the oligonucleotide ligation assay, a rapid and inexpensive test for detection of HIV-1 drug resistance mutations, for non-North American variants. J Acquir Immune Defic Syndr 2008, 48:418-427.

38. Boltz VF, Maldarelli F, Martinson N, Morris L, McIntyre JA, Gray G, Hopley MJ, Kimura T, Mayers $\mathrm{DL}$, Robinson $\mathrm{P}$ et al:: Optimization of allele-specific PCR using patient-specific HIV consensus sequences for primer design. J Virol Methods 2010, 164:122-126.

39. Mutsvangwa J, Beck IA, Gwanzura L, Manhanzva MT, Stranix-Chibanda L, Chipato T, Frenkel LM. Optimization of the oligonucleotide ligation assay for the detection of nevirapine resistance mutations in Zimbabwean human immunodeficiency virus type-1 subtype $\mathrm{C}$. J Virol Methods 2014, 210:36-39.

40. Pillay D, Herbeck J, Cohen MS, de Oliveira T, Fraser C, Ratmann O, Brown AL, Kellam P, PANGEAHIV Consortium: PANGEA-HIV: phylogenetics for generalized epidemics in Africa. Lancet Infect Dis 2015, 15:259-261.

41. Johnson JA, Li JF, Wei X, Lipscomb J, Irlbeck D, Craig C, Smith A, Bennett DE, Monsour M, Sandstrom $\mathrm{P}$ et al.: Minority HIV-1 drug resistance mutations are present in antiretroviral treatment-naïve populations and associate with reduced treatment efficacy. PLoS Med 2008, 5:e158.

42. Peuchant $O$, Thiébaut $R$, Capdepont $S$, Lavignolle-Aurillac $V$, Neau $D$, Morlat $P$, Dabis F, Fleury $H$, Masquelier B, ANRS CO3 Aquitaine Cohort: Transmission of HIV-1 minority-resistant variants and response to first-line antiretroviral therapy. AIDS 2008, 22:1417-1423.

43. Balduin M, Oette M, Däumer MP, Hoffmann D, Pfister HJ, Kaiser R: Prevalence of minor variants of HIV strains at reverse transcriptase position 103 in therapy-naïve patients and their impact on the virological failure. J Clin Virol 2009, 45:34-38.

44. Jakobsen MR, Tolstrup M, Søgaard OS, Jørgensen LB, Gorry PR, Laursen A, Ostergaard L: Transmission of HIV-1 drug-resistant variants: prevalence and effect on treatment outcome. Clin Infect Dis 2010, 50:566-573.

45. Stekler JD, Ellis GM, Carlsson J, Eilers B, Holte S, Maenza J, Stevens CE, Collier AC, Frenkel LM: Prevalence and impact of minority variant drug resistance mutations in primary HIV-1 infection. PLoS One 2011, 6:e28952.

46. Nicot F, Saliou A, Raymond S, Sauné K, Dubois M, Massip P, Marchou B, Delobel P, Izopet J: Minority variants associated with resistance to HIV-1 nonnucleoside reverse transcriptase inhibitors during primary infection. J Clin Virol 2012, 55:107-113.

47. Nishizawa M, Hattori J, Shiino T, Matano T, Heneine W, Johnson JA, Sugiura W: Highly-sensitive allele-specific PCR testing identifies a greater prevalence of transmitted HIV drug resistance in Japan. PLoS One 2013, 8:e83150.

48. Mohamed S, Penaranda G, Gonzalez D, Camus C, Khiri H, Boulmé R, Sayada C, Philibert P, Olive D, Halfon $P$ : Comparison of ultra-deep versus Sanger sequencing detection of minority mutations on the HIV-1 drug resistance interpretations after virological failure. AIDS 2014, 28:1315-1324.

* A French study showing that ultra-deep sequencing reveals additional drug resistance mutations in comparison to Sanger sequencing with significant differences in drug-resistance interpretations. They also indicate that the turnaround time for ultra-deep sequencing is substantially higher.

49. Todesco E, Rodriguez C, Morand-Joubert L, Mercier-Darty M, Desire N, Wirden M, Girard PM, Katlama C, Calvez V, Marcelin AG: Improved detection of resistance at failure to a tenofovir, emtricitabine and efavirenz regimen by ultradeep sequencing. J Antimicrob Chemother 2015, 70:1503-1506.

50. Halvas EK, Wiegand A, Boltz VF, Kearney M, Nissley D, Wantman M, Hammer SM, Palmer S, Vaida $\mathrm{F}$, Coffin JM, Mellors JW: Low frequency nonnucleoside reverse-transcriptase inhibitor-resistant 
variants contribute to failure of efavirenz-containing regimens in treatment- experienced patients. J Infect Dis 2010, 201:672-680.

51. Boltz VF, Bao Y, Lockman S, Halvas EK, Kearney MF, McIntyre JA, Schooley RT, Hughes MD, Coffin JM, Mellors JW, OCTANE/A5208 Team: Low-frequency nevirapine (NVP)-resistant HIV-1 variants are not associated with failure of antiretroviral therapy in women without prior exposure to single-dose NVP. J Infect Dis 2014, 209:703-710.

** An outstanding study showing that minor NNRTI-resistant variants do not always result into a higher risk for virological failure of a first-line NVP-based regimen. A higher risk associated with minor NNRTI-resistant variants was only observed in women previously exposed to a single-dose NVP at delivery.

52. Flys TS, Donnell D, Mwatha A, Nakabiito C, Musoke P, Mmiro F, Jackson JB, Guay LA, Eshleman $\mathrm{SH}$ : Persistence of K103N-containing HIV-1 variants after single-dose nevirapine for prevention of HIV-1 mother-to-child transmission. J Infect Dis 2007, 195:711-715.

53. Delobel P, Saliou A, Nicot F, Dubois M, Trancart S, Tangre P, Aboulker JP, Taburet AM, Molina JM, Massip $\mathrm{P}$ et al.: Minor HIV-1 variants with the K103N resistance mutation during intermittent efavirenz-containing antiretroviral therapy and virological failure. PLoS One 2011, 6:e21655.

54. Charpentier C, Laureillard D, Piketty C, Tisserand P, Batisse D, Karmochkine M, Si-Mohamed A, Weiss L: High frequency of integrase Q148R minority variants in HIV-infected patients naive of integrase inhibitors. AIDS 2010, 24:867-873.

55. Charpentier C, Lee GQ, Rodriguez C, Visseaux B, Storto A, Fagard C, Molina JM, Katlama C, Yazdanpanah $Y$, Harrigan PR, Descamps D: Highly frequent HIV-1 minority resistant variants at baseline of the ANRS 139 TRIO trial had a limited impact on virological response. J Antimicrob Chemother 2015.

56. Pineda-Peña AC, Schrooten Y, Vinken L, Ferreira F, Li G, Trovão NS, Khouri R, Derdelinckx I, De Munter $P$, Kücherer $C$ et al.: Trends and predictors of transmitted drug resistance (TDR) and clusters with TDR in a local Belgian HIV-1 epidemic. PLOS One 2014, 9:e101738.

57. Yang WL, Kouyos RD, Böni J, Yerly S, Klimkait T, Aubert V, Scherrer AU, Shilaih M, Hinkley T, Petropoulos $C$ et al.: Persistence of transmitted HIV-1 drug resistance mutations associated with fitness costs and viral genetic backgrounds. PLoS Pathog 2015, 11:e1004722.

** Study showing that TDR persistence is associated with fitness-cost of drug resistance mutation and its genetic background.

58. Geretti AM, Conibear T, Hill A, Johnson JA, Tambuyzer L, Thys K, Vingerhoets J, Van Delft Y, SENSE Study Group. Sensitive testing of plasma HIV-1 RNA and Sanger sequencing of cellular HIV-1 DNA for the detection of drug resistance prior to starting first-line antiretroviral therapy with etravirine or efavirenz. J Antimicrob Chemother 2014, 69:1090-1097.

* European study showing that sensitive testing did not increase drug resistance levels in therapynaïve HIV-1 patients entering the SENSE trial.

59. Wittkop L, Günthard HF, de Wolf F, Dunn D, Cozzi-Lepri A, de Luca A, Kücherer C, Obel N, von Wyl $V$, Masquelier $B$ et al.: Effect of transmitted drug resistance on virological and immunological response to initial combination antiretroviral therapy for HIV (EuroCoord-CHAIN joint project): a European multicohort study. Lancet Infect Dis 2011, 11:363-371.

60. Wilkin TJ, Goetz MB, Leduc R, Skowron G, Su Z, Chan ES, Heera J, Chapman D, Spritzler J, Reeves JD et al.: Reanalysis of coreceptor tropism in HIV-1-infected adults using a phenotypic assay with enhanced sensitivity. Clin Infect Dis 2011, 52:925-928.

61. Swenson LC, Mo T, Dong WW, Zhong X, Woods CK, Jensen MA, Thielen A, Chapman D, Lewis M, James I et al.: Deep sequencing to infer HIV-1 co-receptor usage: application to three clinical trials of maraviroc in treatment-experienced patients. J Infect Dis 2011, 203:237-245.

62. Swenson LC, Mo T, Dong WW, Zhong X, Woods CK, Thielen A, Jensen MA, Knapp DJ, Chapman D, Portsmouth S et al.: Deep V3 sequencing for HIV type 1 tropism in treatment-naive patients: a reanalysis of the MERIT trial of maraviroc. Clin Infect Dis 2011, 53:732-742.

63. Li JZ, Paredes R, Ribaudo HJ, Svarovskaia ES, Metzner KJ, Kozal MJ, Hullsiek KH, Balduin M, Jakobsen MR, Geretti AM et al.: Low-frequency HIV-1 drug resistance mutations and risk of 
NNRTI-based antiretroviral treatment failure: a systematic review and pooled analysis. JAMA 2011, 305:1327-1335.

64. Cozzi-Lepri A, Noguera-Julian M, Di Giallonardo F, Schuurman R, Däumer M, Aitken S, CeccheriniSilberstein F, D'Arminio Monforte A, Geretti AM, Booth CL et al.: Low-frequency drug-resistant HIV-1 and risk of virological failure to first-line NNRTI-based ART: a multicohort European casecontrol study using centralized ultrasensitive 454 pyrosequencing. J Antimicrob Chemother 2015, 70:930-40.

** European case-control study that confirms the more than double higher risk of virological failure to first-line NNRTI-based regimens.

65. Pou C, Noguera-Julian M, Pérez-Álvarez S, García F, Delgado R, Dalmau D, Álvarez-Tejado M, Gonzalez D, Sayada C, Chueca N et al.: Improved prediction of salvage antiretroviral therapy outcomes using ultrasensitive HIV-1 drug resistance testing. Clin Infect Dis 2014, 59:578-588.

** One of the few studies that investigated the relevance of detecting minority drug-resistant variants in improving genotypic susceptibility scores (GSS) and the prediction of therapeutic outcome in highly-experienced HIV-1 patients.

66. Van Laethem K, De Luca A, Antinori A, Cingolani A, Perna CF, Vandamme AM: A genotypic drug resistance interpretation algorithm that significantly predicts therapy response in HIV-1infected patients. Antivir Ther 2002, 7:123-129.

67. Rhee SY, Liu TF, Kiuchi M, Zioni R, Gifford RJ, Holmes SP, Shafer RW: Natural variation of HIV-1 group $M$ integrase: implications for a new class of antiviral inhibitors. Retrovirology 2008, 5:74.

68. Vingerhoets J, Rimsky L, Van Eygen V, Nijs S, Vanveggel S, Boven K, Picchio G: Pre-existing mutations in the rilpivirine phase III trials ECHO and THRIVE: prevalence and impact on virological response. Antivir Ther 2013, 18:253-256.

69. Mohamed S, Penaranda G, Gonzalez D, Camus C, Khiri H, Boulmé R, Sayada C, Philibert P, Olive D, Halfon P: Comparison of ultra-deep versus Sanger sequencing detection of minority mutations on the HIV-1 drug resistance interpretations after virological failure. AIDS 2014, 28:1315-1324. 
Table 1: HIV-1 drug resistance testing in clinical and epidemiological virology

\begin{tabular}{|c|c|}
\hline Purpose of $t$ & \\
\hline Diagnostics & To detect transmitted drug resistance at diagnosis \\
\hline & To detect (naturally occurring) drug resistance before therapy initiation ${ }^{a}$ \\
\hline & To detect selected drug resistance at therapeutic failure \\
\hline Prognostics & To improve therapeutic outcome \\
\hline
\end{tabular}


Table 2: Characteristics to assess clinical relevance of HIV-1 drug resistance assays

Specifications to assess whether test is fit for purpose: accurate, timely, accessible, understandable, comparable, coherent, complete and reasonably priced

\begin{tabular}{|c|c|c|}
\hline \multicolumn{3}{|c|}{ Advantages and disadvantages of the different technologies } \\
\hline $\begin{array}{l}\text { Population- } \\
\text { based Sanger } \\
\text { sequencing }\end{array}$ & \multicolumn{2}{|c|}{$\begin{array}{l}\text { advantages: turnaround time of a few days, easily implemented into molecular } \\
\text { biology lab, reasonable cost }(\$ 40-80)^{a} \\
\text { disadvantages: low analytical sensitivity for minority variants, no genetic } \\
\text { linkage, no consensus sequence for regions displaying mixtures of indels } \\
\text { (insertions and deletions) }\end{array}$} \\
\hline $\begin{array}{l}\text { Mutation- } \\
\text { specific } \\
\text { technology }\end{array}$ & \multicolumn{2}{|c|}{$\begin{array}{l}\text { advantages: high analytical sensitivity for minority variants, shortest } \\
\text { turnaround time, easiest to be implemented into molecular biology lab, easy } \\
\text { interpretation, qualitative and quantitative results, relative inexpensive }(\$ 40)^{\text {a }} \text {, } \\
\text { suitable for large batches } \\
\text { disadvantages: only sentinel positions, lower analytical specificity, no genetic } \\
\text { linkage }\end{array}$} \\
\hline & \multicolumn{2}{|c|}{$\begin{array}{l}\text { advantages: high analytical sensitivity for minority variants, genome-wide } \\
\text { approach, potential of genetic linkage, qualitative and quantitative results, low } \\
\text { reagent costs }(\$ 100)^{\text {a }} \text {, suitable for large batches } \\
\text { disadvantages: sequencing errors, turnaround time, need for multidisciplinary } \\
\text { team (wet-lab and in-silico), high indirect costs (equipment, labor) }\end{array}$} \\
\hline Common & \multicolumn{2}{|c|}{$\begin{array}{l}\text { disadvantages: errors during template enrichment by RT-PCR (mutation, } \\
\text { recombination), false negative results due to genetic variability at } \\
\text { annealing/hybridization sites, resampling and stochastic amplification at low } \\
\text { template concentrations }\end{array}$} \\
\hline \multicolumn{3}{|c|}{ Evaluation of performance characteristics: } \\
\hline & \multicolumn{2}{|l|}{ Analytical characteristics } \\
\hline $\begin{array}{l}\text { Approved } \\
\text { commercial } \\
\text { assay }^{\text {b }}\end{array}$ & $\begin{array}{l}\text { Measurement range, precision, trueness, } \\
\text { linearity }{ }^{d}\end{array}$ & Reference range $^{d}$ \\
\hline In-house assay ${ }^{c}$ & $\begin{array}{l}\text { Detection limit, quantification limit } \\
\text { robustness, analytical sensitivity and } \\
\text { specificity, selectivity }\end{array}$ & Diagnostic sensitivity and \\
\hline \multicolumn{3}{|c|}{$\begin{array}{l}{ }^{a} \text { Reagent cost for monitoring protease and reverse transcriptase with in-house methods [35,69]. }{ }^{b} \\
\text { Characteristics that should be evaluated for the verification of an approved commercially available } \\
\text { assay. }{ }^{c} \text { Characteristics that additionally should be evaluated for the validation of an in-house assay. } \\
\text { Characteristics that should additionally be evaluated for quantitative assays. }\end{array}$} \\
\hline
\end{tabular}


Figure 1: Consequences of applying various methods for genotypic drug resistance testing.

The consensus sequence obtained with population-based Sanger sequencing from a patient infected with HIV-1 CRF02_AG and failing a combination of tenofovir (TDF), lamivudine (3TC) and nevirapine (NVP), displays a mixture at reverse transcriptase (RT) position 65 (associated with TDF resistance) and a pure mutation at RT position 181 (associated with NVP resistance). An env consensus sequence cannot be obtained for the region immediately upstream of the V3 loop due to mixtures of viral variants with and without a deletion.

Mutation-specific assays do not monitor the rare K65R position in RT and the V3 loop, but viral variants displaying the 3TC mutation M184V are detected at a low frequency (5\%). Ultra-deep sequencing gives in-depth information on the resistance profile within the genetic region covered, quantifying 50\% K65R, 100\% Y $181 \mathrm{C}$ and 5\% M184V. In an ultra-wide approach of next generation sequencing, more regions are covered, but at a lower sensitivity and therefore missing the M184Vcontainting variants. 


\section{viral target \\ codon}

Sanger sequencing

mutation-specific assay

ultra-deep sequencing

ultra-wide sequencing reverse transcriptase

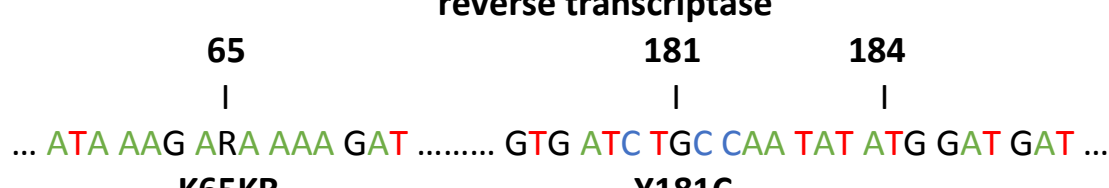

Y181C

$100 \%$ Y $181 \mathrm{C} \quad 5 \% \mathrm{M} 184 \mathrm{~V}$
100\% Y181C $5 \%$ M184V

... ATA AAG AGA AAA GAT ... ... GTG ATC TGC CAA TAT ATG GAT GAT ... ... ATA AAG AGA AAA GAT ... ... GTG ATC TGC CAA TAT ATG GAT GAT ... ... ATA AAG AAA AAA GAT ... ... GTG ATC TGC CAA TAT ATG GAT GAT ... ... ATA AAG AGA AAA GAT ... ... GTG ATC TGC CAA TAT ATG GAT GAT ... ... ATA AAG AGA AAA GAT ... ... GTG ATC TGC CAA TAT ATG GAT GAT ...

... ATA AAG AAA AAA GAT ... ... GTG ATC TGC CAA TAT ATG GAT GAT ... ... ATA AAG AAA AAA GAT ... ... GTG ATC TGC CAA TAT ATG GAT GAT ... ... ATA AAG AAA AAA GAT ... ... GTG ATC TGC CAA TAT ATG GAT GAT ... ... ATA AAG AAA AAA GAT ... ... GTG ATC TGC CAA TAT GTG GAT GAT ... ... ATA AAG AGA AAA GAT ... ... GTG ATC TGC CAA TAT ATG GAT GAT ... $50 \%$ K65R

... ATA AAG AGA AAA GAT ... ... GTG ATC TGC CAA TAT ATG GAT GAT ... ... ATA AAG AGA AAA GAT ... ... GTG ATC TGC CAA TAT ATG GAT GAT ... ... ATA AAG AAA AAA GAT ... ... GTG ATC TGC CAA TAT ATG GAT GAT ... ... ATA AAG AAA AAA GAT ... ... GTG ATC TGC CAA TAT ATG GAT GAT ... $50 \%$ K65R

\author{
V3 loop \\ 1 \\ I \\ ... NNN TTT AAT GGA ACA GGG CYA TGC CAG ... \\ CCR5
}

not performed

not performed

... ACG TTT AAT GGA ACA GGG CTA TGC CAG ... ... - - - TTT AAT GGA ACA GGG CCA TGC CAG ... ... ACG TTT AAT GGA ACA GGG CTA TGC CAG ... ... - . - TTT AAT GGA ACA GGG CCA TGC CAG ...

CCR5 